\title{
Study on Transportation Planning System: Based on Case-Based Reasoning
}

\author{
Nie Bin ${ }^{1, *}$, Pi $\mathrm{Li}^{2}$, Fan Zhengwei ${ }^{1}$ and Yang Huiyuan ${ }^{3}$ \\ ${ }^{1}$ Bengbu Automobile NCO College, Bengbu, Anhui, 233011, P.R. China; ${ }^{2}$ National University of Defense Technology, \\ Changsha, Hunan, 410073, P.R. China; ${ }^{3}$ Anhui University of Finance \& Economics, Bengbu, Anhui, 233011, P.R. \\ China
}

\begin{abstract}
This paper analyses the flow chart of the transportation planning system based on Case-based Reasoning, and discusses the framework knowledge for representing a transportation plan. Moreover, it puts forwards a retrieval algorithm model for similar cases.
\end{abstract}

Keywords: Case retrieval, case-based reasoning, framework knowledge representation, road transportation plan.

\section{INTRODUCTION}

Transportation plan is the base and fundament for the armed force to organize transportation support. Currently, the methods we plan transportation are limited to traditional experiences, which have insufficient scientific theories and modern technologies supports and stand on a low level of automating and intellectualizing progress. This paper, by taking road transportation planning as the example, studies how to apply CBR for planning transportation support and puts forwards the key techniques and operating mechanisms of the expected systems (based on CBR) which can create transportation plans.

\section{THE PLANNING PROCEDURE BASED ON CBR}

The planning procedure based on CBR will integrate the advantages of automatically generating and intelligently assessing a plan, which can significantly improve the efficiency and quality of the work of transportation planning [1]. The specific procedure can be introduced as the following 4 steps (see Fig. 1):

(1) Extract the features of the target issue: according to the features of the expected plan, extract its features and the relationships between these features. Take the road transportation supporting plan as example, its initial features include the type, major contents modules and so on.

(2) Retrieve and extract the cases: according to abovementioned features, use specific retrieval algorithm to search corresponding similar cases from the case base.

(3) Assess and modify the selected cases: from the cases been searched out, find out some cases or their combination which are most alike the target issue, then by assessing and modifying them to answer the requirements of planning.

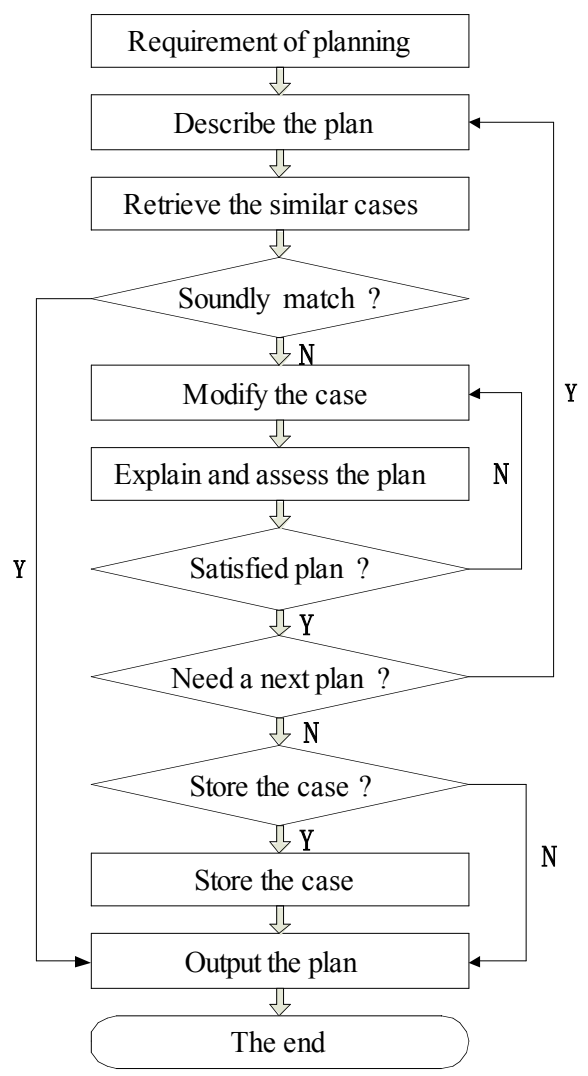

Fig. (1). The flow chart of transportation planning system.

(4) Store the new cases: the new cases produced in the process of planning can be added into the case base to amplify the number of templates for later planning.

\section{CASE REPRESENTATION OF TRANSPORTATION PLAN}

Case representation is one way to represent knowledge, it has direct relationship to the efficiency of reasoning and the quality of solutions. In the current knowledge system, the 


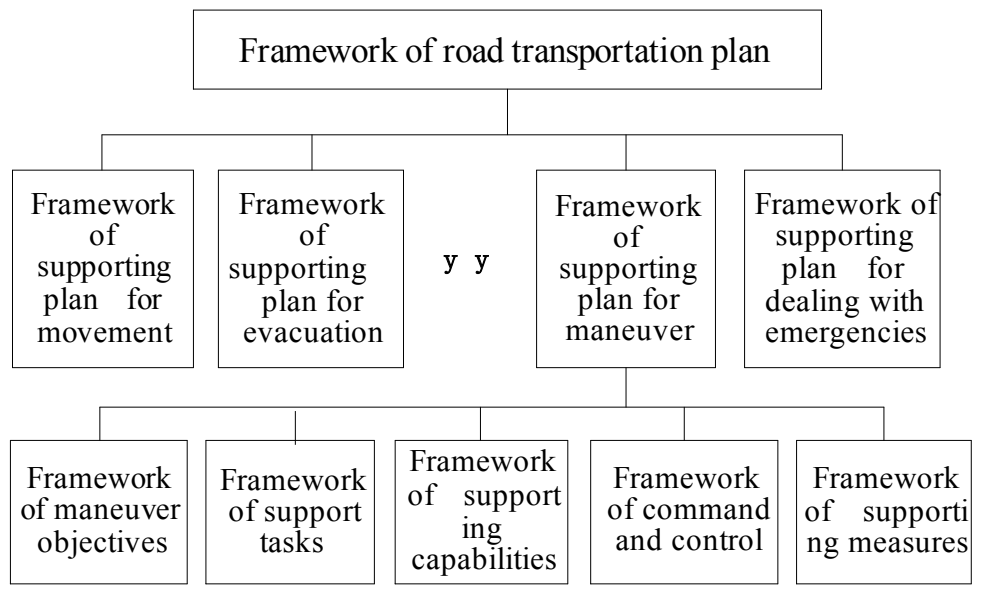

Fig. (2). Framework network structure of transportation plans.

common ways of representing knowledge are: generating, framework, predication, semantic network, object-oriented structured representation and so on. Because framework is a more efficient way to representing structured knowledge, the framework net can make a complex issue easier, furthermore, the transportation plan has a distinct character of structure, so we chose framework to represent transportation plan. Its framework network structure is as Fig. (2). The relationships between these frameworks are mainly longitudinally, and the total framework system shows a layer hierarch network structure.

According to framework theory, a framework is a data structure for describing an object; it is constructed by a name and a series of slots [2]. Every slot has more than one facets. A slot value stands for one attribute of the object, and a facet is used to describe the character of a slot from specific aspect. Every framework is an unique knowledge unit, augmenting, deleting, and modifying it will not influence other frameworks, that is to say, the work of representing by framework shows some features of modularity. When we reasoning a framework, we chose candidate frameworks from the data in the context, and by ways of querying, defaulting, inheriting, adding and so on to acquire the attribute value. For example, the framework of a road transportation plan can be described in Table $\mathbf{1}$.

\section{ALGORITHM MODEL FOR CASE MATCHING}

The purpose of case retrieving is to promptly and precisely search some plans which are the most similar to target transportation plan from the case base. For this purpose, the numbers of retrieval results must be as minimum as possible, and the cases searched out will have the highest similarity to target issues. So, the retrieval tactic is very important [3].

\subsection{Case Retrieval Algorithm Based on Priority Degree}

Transportation plan is a kind of forecasting plan, it involves so much elements and has the characteristics of nonlinearity and fuzziness. To measure the similarity between the elements in this kind of plan, the similarity model based on priority degree can be used.
Table 1. Framework representing of road transportation supporting plan.

Framework name: < road transportation supporting plan for operations >

Subordinate framework name 1: < transportation supporting >

Slot name 11: Petrol supports, facet 111: supported unit, facet 112: Supporting place facet 113:supporting approach

Slot name 12:load and unload, facet 121:loading place, facet 122: Unloading place facet 123:time for loading

Subordinate framework name 2: < supporting capability allocation >

Slot name 21:basic transportation capability, facet 211:vehicles, facet 212: percentage ratio to the total transportation capability

Slot name 22:attached transportation capability, facet 221:vehicles, facet 222:percentage ratio to the total transportation capability

The attribute values can be represented by vectors to facilitate searching for vecto spaces and to realize case retrieving and matching.

Assume case base $e=\left\{e_{1}, e_{2}, \cdots, e_{k}, \cdots e_{m}\right\}$, there into $e_{k}=\left(f_{k 1}, f_{k 2}, \cdots, f_{k j}, \cdots, f_{k n}\right), f_{k j}$ stands for the $j$ attribute of case $e_{k}$, the weight allocation for each attributes is $W=\left(w_{1}, w_{2}, \cdots, w_{j}, \cdots w_{n}\right), 0 \leq w_{j} \leq 1$, and $\sum_{j=1}^{n} w_{j}=1$.

Assume $e_{p}=\left(f_{P 1}, f_{P 2}, \cdots, f_{P j}, \cdots, f_{P n}\right)$ and $e_{q}=\left(f_{q 1}\right.$, $\left.f_{q 2}, \cdots, f_{q j}, \cdots, f_{q n}\right)$ are two random different plans in the case base, $e_{0}=\left(f_{01}, f_{02}, \cdots, f_{0 j}, \cdots, f_{0 n}\right)$ is the target plan.

Define the distance between $f_{p j} 0 \mathrm{e} f_{0 j} \quad$ is $d\left(f_{p j}, f_{0 j}\right)=\left|f_{p j}-f_{0 j}\right|$, the distance between $f_{q j}$ and $f_{0 j}$ is $d\left(f_{q j}, f_{0 j}\right)=\left|f_{q j}-f_{0 j}\right|$. 
Table 2. The attributes value of the cases and target plan.

\begin{tabular}{|c|c|c|c|c|}
\hline Attributes & Case $e_{1}$ & Case $e_{2}$ & Case $e_{3}$ & Case $e_{0}$ \\
\hline Predicted petrol for transportation $(\mathrm{t})$ & 136 & 124 & 170 & 142 \\
\hline Predicted ammunition for transportation ( $\mathrm{t}$ ) & 593 & 550 & 654 & 610 \\
\hline Transporter & 130 & 124 & 148 & 140 \\
\hline
\end{tabular}

Define the priority degree of $f_{p j}$ to $f_{q j}$ and $f_{0 j}$ is :

$$
r_{p q}^{j}=1-d\left(f_{p j}, f_{0 j}\right) /\left[d\left(f_{p j}, f_{0 j}\right)+d\left(f_{q j}, f_{0 j}\right)\right] r_{p q}^{j} \in(0,1)
$$

The bigger the $r_{p q}^{j}$ value, the more similarity $f_{q j}, f_{p j}$ to $f_{0 j}$. Therefore the priority matrix $R^{j}$ to the $j$ attribute can be got:

$$
R^{j}=\left[\begin{array}{cccc}
r_{11}^{j} & r_{12}^{j} & \cdots & r_{1 n}^{j} \\
r_{21}^{j} & r_{22}^{j} & \cdots & r_{2 n}^{j} \\
& \cdots \cdots & & \\
r_{n 1}^{j} & r_{n 2}^{j} & \cdots & r_{n n}^{j}
\end{array}\right]
$$

Set a threshold value $\lambda$ to priority matrix $R^{j}$, then can get $\lambda$-matrix $R_{\lambda}^{j}=\left(r_{i k}^{j}\right)_{\lambda}$, there into $\lambda \in[0,1]$, $\left(r_{i k}^{j}\right)_{\lambda}=\left\{\begin{array}{c}1,\left(r_{i k}^{j}\right)_{\lambda} \geq \lambda \\ 0,\left(r_{i k}^{j}\right)_{\lambda}<\lambda\end{array}\right.$

If $R_{\lambda}^{j}$ has a row whose elements are all " 1 " except for the elements on the diagonals, then the attributes of the cases corresponding to this row are the first sort of similar objects, it can be numbered " 1 ". Thereafter get rid of the rows and lines corresponding to the first sort of similar objects, another priority matrix can be got, then lower the threshold value $\lambda$ and continue the above procedure. when lowering $\lambda$ from 1 to 0 , a series of similar objects are got, they are in ascending order of number, and the smaller their numbers, the higher their similarities. The number of $m$ cases can be gathered to be a number set:

$$
T=\left\{t_{1 j}, t_{2 j}, \cdots, t_{m j}\right\},
$$

$t_{k j}$ stands for the similarity number of the $j$ attribute of the $\mathrm{k}$ case to the new issue, by this we can get the priority number of case $\mathrm{k}$ compared with other cases:

$$
t_{k}=\sum_{k=1}^{m} \omega_{j} \cdot t_{k j},
$$

and the most suitable case is the case whose $t_{k}$ value is smallest.

\subsection{Application Example}

Take road transportation plan as the example, some characteristic data of case base and the target plan are as Table 2.

Assume the weight of the transporter, petrol, and ammunition are respectively: $0.4,0.3,0.3$, then for the part of predicting the quantity of petrol for transportation, the 3 case are sequenced in their similarity to the target plan by the follow steps:

(1) get the semantic distance

It can be deduced from the data in Table 2 and the formula 6 that: $d\left(f_{11}, f_{01}\right)=|136-142|=6$, likewise, $d\left(f_{21}, f_{01}\right)=|124-142|=18$, $d\left(f_{31}, f_{01}\right)=|170-142|=28$.

(2) construct priority matrix and sequence them

$$
r_{12}^{1}=1-d\left(f_{11}, f_{01}\right) /\left[d\left(f_{11}, f_{01}\right)+d\left(f_{21}, f_{01 j}\right)\right]=0.75,
$$

likewise can get the value of $r_{p q}^{1}$, there into $p, q=1,2,3$. The corresponding priority matrix is: $R^{1}=\left[\begin{array}{ccc}1 & 0.75 & 0.82 \\ 0.25 & 1 & 0.61 \\ 0.18 & 0.39 & 1\end{array}\right], \quad$ take $\quad \lambda=0.7, \quad$ and $R_{0.7}^{1}=\left[\begin{array}{lll}1 & 1 & 1 \\ 0 & 1 & 0 \\ 0 & 0 & 1\end{array}\right]$. In the first row of $R_{0.7}^{1}$, all elements are " 1 ", then for the part of predicting the quantity of petrol for transportation, case $c_{1}$ is the most similar case to required plan. Get rid of the first row and the first line, following matrix is got:

$$
R^{11}=\left[\begin{array}{cc}
1 & 0.61 \\
0.39 & 1
\end{array}\right] \text {. }
$$

Likewise, take the threshold value $\lambda$ as 0.6 , the sequence for the other 2 cases are created. Then the similarity sequence of the cases in case base to the required case from the aspect of the first attribute can be got: $T_{1}=\{1,2,3\}$. In like manner can get the similarity sequence of the other attribute to the new issue, they are respectively: $T_{2}=\{1,3,2\}$, $T_{3}=\{3,1,2\}$. Lastly, we can get the priority sequence number of each case to the required plan: 


$$
\begin{aligned}
& t_{1}=\sum_{k=1}^{3} \omega_{j} \cdot t_{k j}=1 \times 0.3+1 \times 0.3+3 \times 0.4=1.8 \\
& t_{2}=1.9, t_{3}=2.3 . t_{1} \text { is the smallest one, so case } 1 \text { is }
\end{aligned}
$$
the best case.

Because there are differences between the case searched out and the new issue, so modifying is needed for adapting to the required new plan. We use the strategy of plan transformation based on framework to modify the case, use the most similar case as the basic framework to compare its subordinate frameworks with the required plan's described value, and find their similarities and differences, then start to modify and adjust the case. If the case have deficiencies, use some part of the other cases to complement it, if it still cannot answer the requirement, the planning staffs will adjust it interactively. The modified plan will be accessed by personcomputer interactive system, until we get a satisfied new plan. If the new plan is deemed to have reference value, it can be added into the case base for future use.

\section{CONCLUSION}

As a reasoning method closer to human thinking habit, CBR and its application have been focused on designing and management area. Introducing CBR into road transportation planning and constructing an automatic transportation plan system will help to plan the road transportation more scientifically and intelligently.

\section{CONFLICT OF INTEREST}

The authors confirm that this article content has no conflict of interest.

\section{ACKNOWLEDGEMENTS}

Declared none.

\section{REFERENCES}

[1] D.W. Aha, I. Watson, "Case-Based Reasoning Research and Development", Springer, pp. 35-42, 2001.

[2] W. Hu, S. Wang, W. Pan, "Research and implement of case-based reasoning in online logistics resource decision," Journal of Wuhan University of Technology, vol. 27, pp. 581-584, Aug. 2003.

[3] J.K. Lee, J.K. Kim, "A case-based reasoning approach for building a decision model,” Expert Systems, vol. 19, pp.123-135, Jun. 2002.

Received: June 10, 2015

Revised: July 29, 2015

Accepted: August 15, 2015

(C) Bin et al.; Licensee Bentham Open.

This is an open access article licensed under the terms of the (https://creativecommons.org/licenses/by/4.0/legalcode), which permits unrestricted, noncommercial use, distribution and reproduction in any medium, provided the work is properly cited. 